\title{
When Musical Accompaniment Allows the Preferred Spatio- Temporal Pattern of Movement
}

\section{()(1) $\odot \Theta$}

\section{Authors}

Analina Emmanouil ${ }^{1}$, Elissavet Rousanoglou ${ }^{1}$, Anastasia Georgaki ${ }^{2}$, Konstantinos D. Boudolos ${ }^{1}$

\section{Affiliations}

1 National and Kapodistrian University of Athens, Faculty of Physical Education and Sport Science, Department of Sport Medicine and Biology of Exercise, Sport Biomechanics Lab, Daphne, Greece

2 National and Kapodistrian University of Athens, Department of Music Studies, Athens, Greece

\section{Key words}

tempo, pulse clarity, rhythmic strength, propensity to move, postural sway

received 23.02.2021

revised 04.05 .2021

accepted 11.05.2021

\section{Bibliography}

Sports Medicine International Open 2021; 5: E81-E90

DOI 10.1055/a-1553-7063

ISSN 2367-1890

(c) 2021. The Author(s).

This is an open access article published by Thieme under the terms of the Creative Commons Attribution-NonDerivative-NonCommercial-License, permitting copying and reproduction so long as the original work is given appropriate credit. Contents may not be used for commercial purposes, or adapted, remixed, transformed or built upon. (https://creativecommons. org/licenses/by-nc-nd/4.0/)

Georg Thieme Verlag KG, Rüdigerstraße 14,

70469 Stuttgart, Germany

\section{Correspondence}

Dr. Elissavet Rousanoglou

National \& Kapodistrian University of Athens, Faculty of Physical Education and Sport Science, Department of Sport Medicine \& Biology of Exercise, Sport Biomechanics Lab, School of Physical Education \& Sport Science,

Ethnikis Antistasis 41

172-37 Athens

Greece

Tel.: + 302107276090, Fax: + 302107276090

erousan@phed.uoa.gr
Supplementary Material is available under https://doi. org/10.1055/a-10.1055/a-1553-7063

\begin{abstract}
A musical accompaniment is often used in movement coordination and stability exercise modalities, although considered obstructive for their fundament of preferred movement pace. This study examined if the rhythmic strength of musical excerpts used in movement coordination and exercise modalities allows the preferred spatio-temporal pattern of movement. Voluntary and spontaneous body sway (70 s) were tested ( $\mathrm{N}=20$ young women) in a non-musical (preferred) and two rhythmic strength (RS) musical conditions (Higher:HrRS, Lower:LrRS). The center of pressure trajectory was used for the body sway spatio-temporal characteristics (Kistler forceplate, $100 \mathrm{~Hz}$ ). Statistics included paired t-tests between each musical condition and the non-musical one, as well as between musical conditions $(p \leq 0.05)$. Results indicated no significant difference between the musical and the non-musical conditions ( $p>0.05)$. The HrRS differed significantly from LrRS only in the voluntary body sway, with increased sway duration $(p=0.03)$, center of pressure path $(p=0.04)$ and velocity $(p=0.01)$. The findings provide evidence-based support for the rhythmic strength recommendations in movement coordination and stability exercise modalities. The HrRS to LrRS differences in voluntary body sway most possibly indicate that low-frequency musical features rather than just tempo and pulse clarity are also important.
\end{abstract}




\section{Introduction}

Musical accompaniment is documented to enhance performance in endurance exercise modalities [1-3] as well as in muscular strength ones [4]. However, in movement coordination and stability (MCS) exercise modalities (i. e., Pilates), a musical accompaniment is unadvised as obstructive for their fundament of preferred movement pace $[5,6]$. The inherent exercise description according to the breathing pattern in MCS modalities [5, 6] postulates the notion of preferred pace during learning and performing the movement. The notion that a musical accompaniment may potentially disrupt rather than benefit the efficiency of performance rests in previous studies. Specifically, music may potentially impair postural tasks [7-9] and distract concentration during coordination and precision tasks [10]. It may also have a musical genre-specific effect on the movement's amount and fluidity [11] as well as its perceived easiness, preciseness, and rhythmicity [12].

The potential incompatibility of musical accompaniment with the preferred movement pace is reasoned on the participant's regulation away from his/her natural periodic pace $[13,14]$ or movement duration [10]. However, the association of music with pleasure [15] motivates its use in MCS exercise modalities despite its potential contrast to the fundament of one's preferred pace. As such, a musical stimulus of low tempo ( $<100 \mathrm{bpm}$ : beats per minute), and low pulse clarity (music without a strong beat) is recommended in relevant textbooks [16]. However, such suggestions appear extrapolated rather than evidence-based, reasoned on the relationship between the propensity to move and rhythmic strength descriptors of the musical signal (i. e., tempo, pulse clarity, event density, spectral flux, low energy) [17-19]. The response of the autonomic nervous system to tempo, which is suppressed parasympathetic and increased sympathetic activation in tempi higher than the normal heart rate during long-lasting passive music listening, also explains the slow tempo recommendation in MCS exercise modalities [20]. However, to the best of our knowledge, there is no previous study documenting that the musical accompaniment used in MCS exercise modalities does allow the fundament of one's preferred movement pace, or does not influence the preferred spatio-temporal movement pattern.

Similar to Burger et al. [14] and Madison et al. [21], we aimed at designing a study of the higher possible ecological validity, in so far as this is allowed in a laboratory situation. To this end, we chose real pre-existing musical excerpts among those used in MCS exercise modalities, accepting the downside [21] that their propensity-tomove properties [22] were not tested. However, this approach made it possible to present the participants with the kind of music that they were familiar with. To further enhance the external validity of our study, the voluntary and spontaneous body sway were selected as movement tasks, reflecting the whole body weight shifting and static balance tasks commonly employed in MCS exercise modalities [23,24]. Furthermore, the body sway tasks allowed the comparison with previous studies addressing the musical stimulus effect on voluntary [25] or spontaneous [7, 8, 25-29] body sway. The voluntary body sway was of particular interest, because despite the need for voluntary rather than spontaneous body sway in daily activities, the relevant studies appear limited to the influence of discrete (i. e., metronome beats) rather than continuous auditory stimuli [25].

Thus, the purpose of the study was to examine if pre-existing musical excerpts among those commonly used to accompany MCS exercise modalities allow the preferred spatio-temporal pattern of movement during voluntary and spontaneous body sway. Because the preferred movement pace is a fundament of such modalities, we hypothesized that the selected musical stimuli would not significantly alter the spatio-temporal characteristics of the voluntary and spontaneous body sway when compared to a non-musical stimulus condition.

\section{Materials and Methods}

\section{Participants}

Twenty healthy women participated in the study ( $\vee$ Table 1 shows their characteristics and the inclusion criteria [28, 30-33]). The uHear application (a pure-tone hearing sensitivity test, which runs on iOS devices, iPod, iPhone, and iPad) was used to verify their normal and symmetrical hearing. The study meets the ethical standards described by Harriss et al. [34] and was approved by the University Bioethics Committee. All participants signed informed consent.

\section{Musical stimuli}

As recommended for MCS exercise modalities [16], two musical pieces within the slow to medium tempo range [19] were selected ( $\vee$ Fig. 1). The pieces were chosen from a list suggested by experienced instructors, who perceptually discriminated them into higher (HrRS) and lower (LrRS) rhythmic strength (RS) (no specific criteria were provided). They were chosen from among instrumental ones to avoid any physiological [3], mental [35], attentional [36], or emotional [37] influence due to the lyrics. A musical signal analysis (

- Table 1 Characteristics (mean \pm SD) of the participants $\left(n=20\right.$ women $\left.{ }^{*}\right)$ and their inclusion criteria.

\begin{tabular}{|l|l|l|}
\hline Characteristics & Mean \pm SD & Inclusion criteria ${ }^{* *}$ \\
\hline Age $(\mathrm{yrs})$ & $26.0 \pm 4.2$ & Gender (only women), age range (from 20 to 30 yrs) [30], moderate physical activity [31]. \\
\hline Body height $(\mathrm{m})$ & $1.72 \pm 0.06$ & $\begin{array}{l}\text { Anthropometrics [32]: body height range (from 1.60 to 1.75 m), within the normal range of body } \\
\text { mass index (from 18.5 to } 24.9 \mathrm{~kg} / \mathrm{m}^{2} \text { ). }\end{array}$ \\
\hline Body mass $(\mathrm{kg})$ & $68.1 \pm 10.6$ & No musical training or experience [28]. \\
\hline Body mass index $\left(\mathrm{kg} / \mathrm{m}^{2}\right)$ & $21.2 \pm 2.0$ & No visual, vestibular, auditory, musculoskeletal or neurological disorder [33]. \\
\hline $\begin{array}{l}\text { "A sample of } 20 \text { subjects was considered adequate for an empirically estimated "smallest expected effect" of } \mathrm{d}=0.65 \text { to be detected in "musical } \\
\text { versus non-musical" comparisons with a power of at least } 80 \% \text {, assuming a two-tailed test at } \alpha=0.05 \text { (details in the "Sample Power Analysis" } \\
\text { supplementary material). }{ }^{*} \text { "Inclusion criteria aimed to eliminate potential confounding factors. }\end{array}$ \\
\hline
\end{tabular}


Total Stimulus Duration
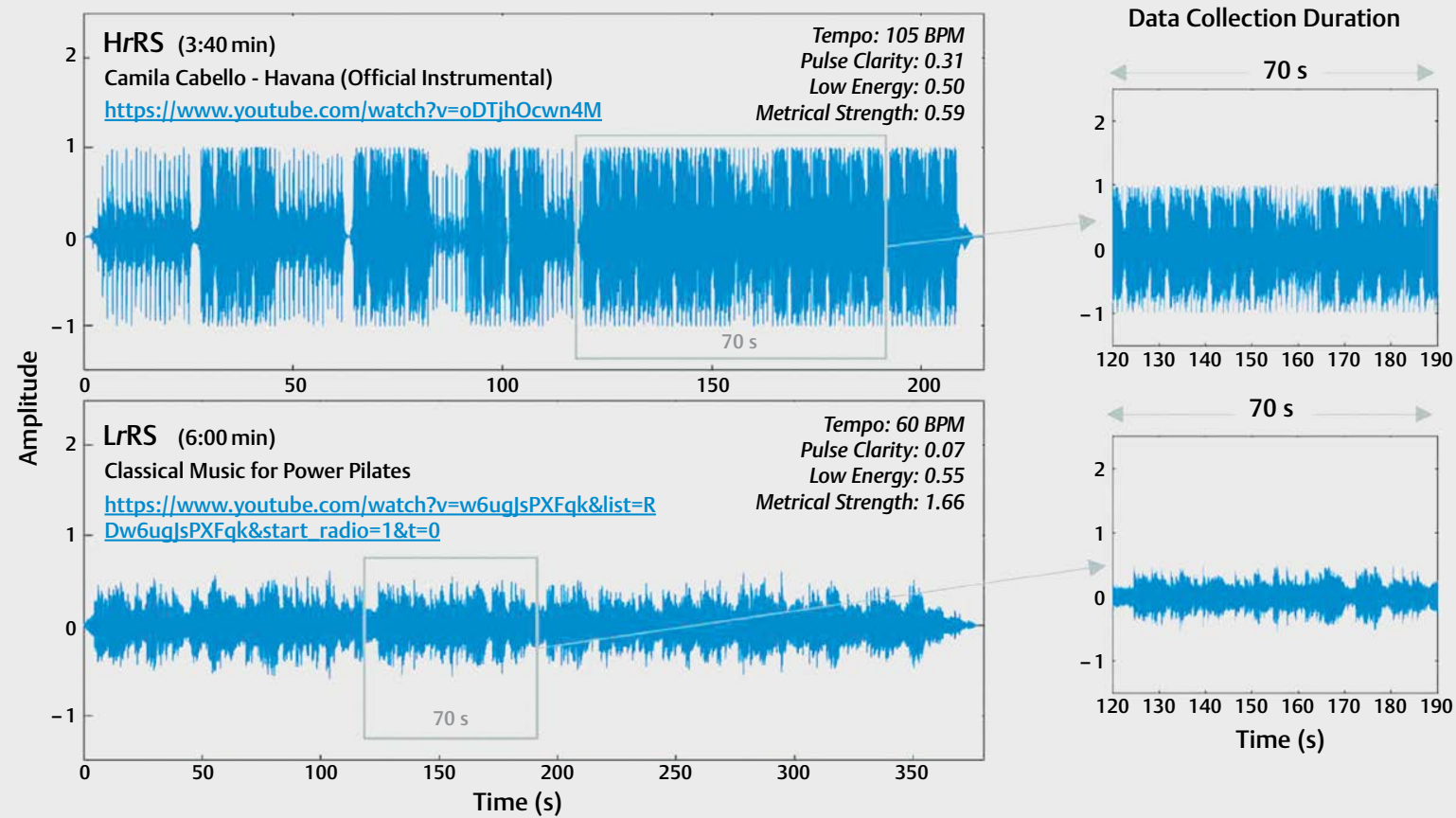

Bottom: Spectrograms

\section{$(0-4000 \mathrm{~Hz})$ \\ Total Stimulus Duration}

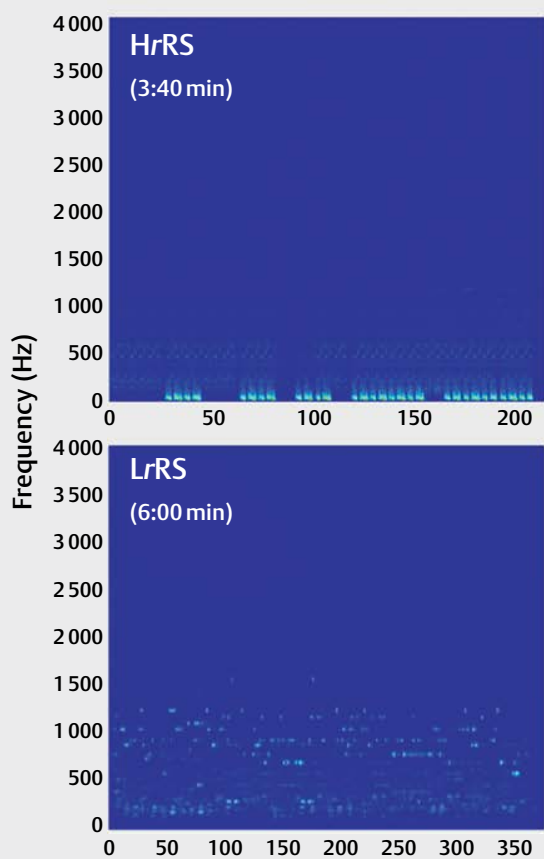

$(0-4000 \mathrm{~Hz})$

Data Collection Duration

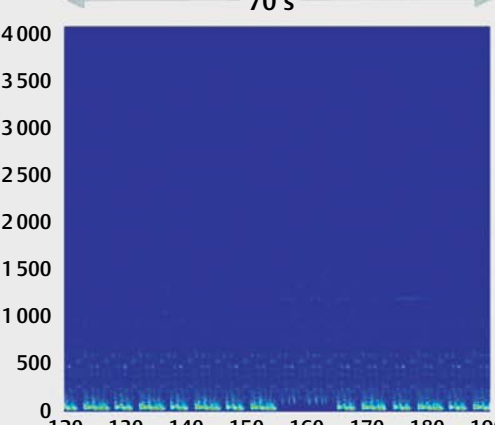

$\begin{array}{lllllllll}0 & 120 & 130 & 140 & 150 & 160 & 170 & 180 & 190\end{array}$

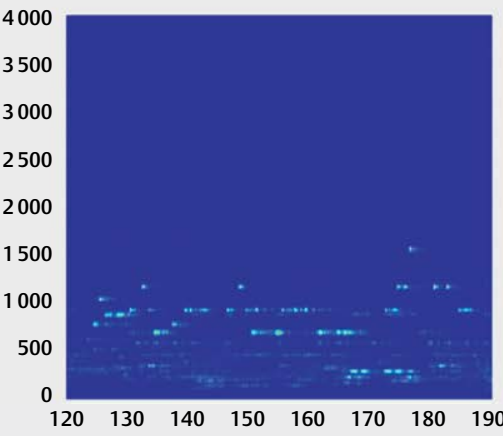

$(0-200 \mathrm{~Hz})$

Representative Duration

$5 \mathrm{~s}$

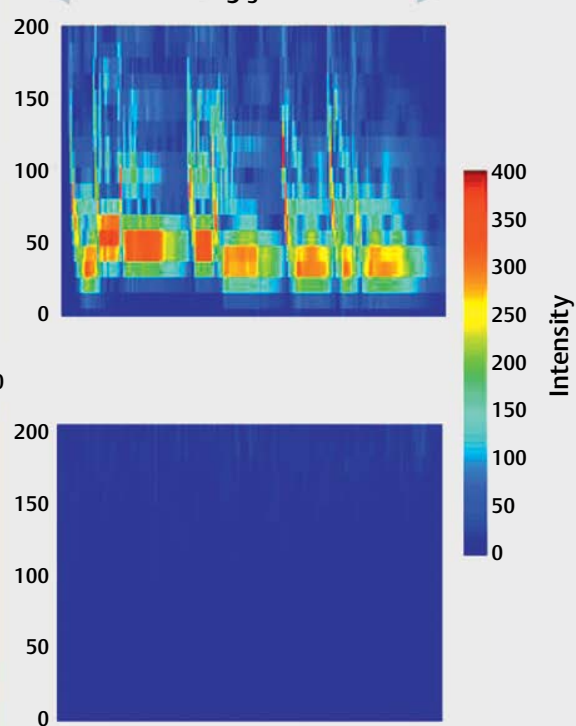

Time (s)

Fig. 1 Waveforms (top) and spectrograms (bottom) of the higher (HrRS) and the lower (LrRS) rhythmic strength (RS) musical stimuli. The information embedded in the waveform panels indicate the values of the musical features associated with RS, extracted through musical analysis (MIRtoolbox 1.7 software [38]). Details of the relevant MIRtoolbox routines used to extract the specific musical features are provided in the supplementary material under the title MIRtoolbox signal analysis. Top left: Total duration of each musical stimulus. Top right: Data collection duration (70 s) of each musical stimulus. Bottom left: Total duration waveform of each musical stimulus across the $0-4000 \mathrm{~Hz}$ frequency band. Bottom middle: Waveform of each musical stimulus across the $0-4000 \mathrm{~Hz}$ frequency band for the data collection duration ( $70 \mathrm{~s})$. Bottom right: Indicative $5 \mathrm{~s}$ waveform of each musical stimulus enlarged for the $0-200 \mathrm{~Hz}$ frequency band (the frequency band associated with the affordance of a musical stimulus to affect body movement $[13,46,47,56]$ ) illustrating the greater flux (intensity fluctuation) in the HrRS than the LrRS musical stimulus. 
HrRS

(tempo match: $100 \%$, test value $=105 \mathrm{bpm}$ )
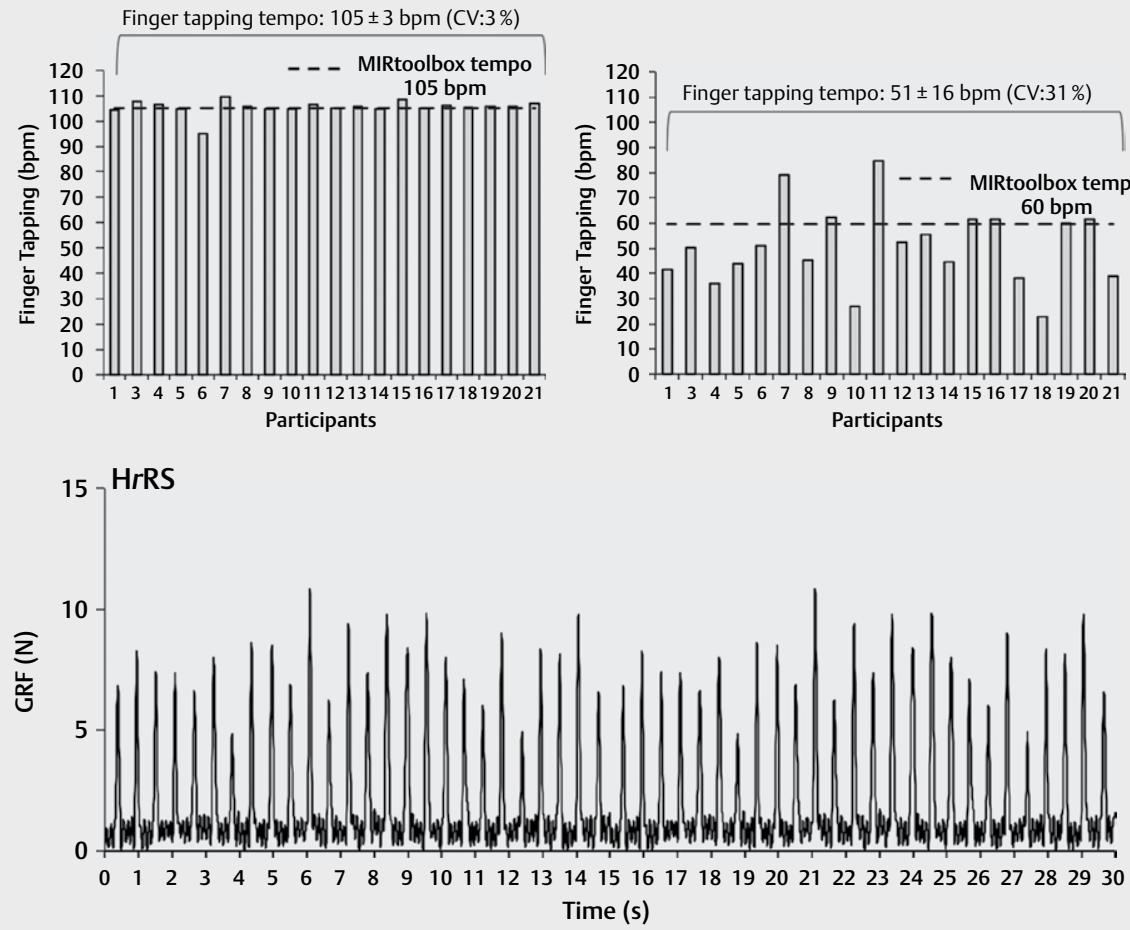

LrRS

(tempo match: $86 \%$, test value $=60 \mathrm{bpm}$ )

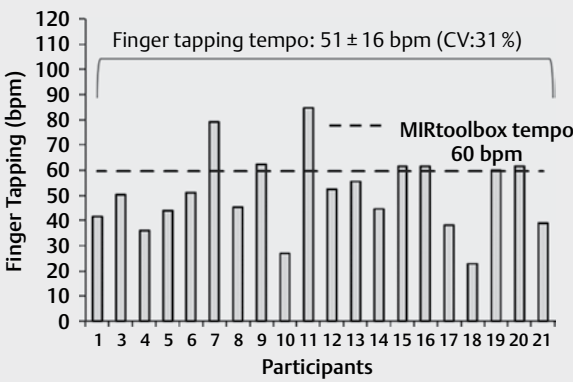

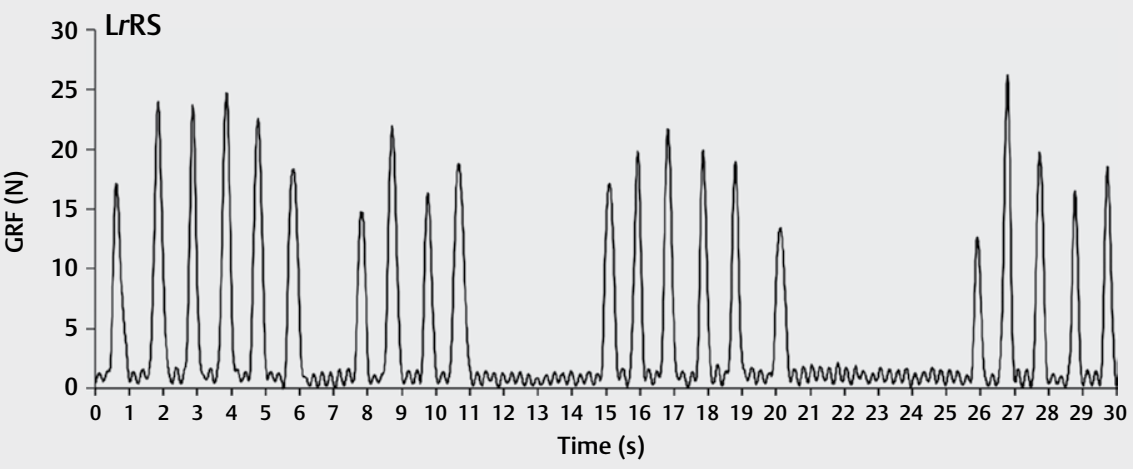

- Fig. 2 Top: Finger tapping tempo in the higher (HrRS) (left) and the lower (LrRS) (right) rhythmic strength musical stimuli. The mean \pm SD as well as the percentage coefficient of variability (CV\%: (SD/mean) $\times 100)$ of the finger tapping tempo are noted. The tempo match between finger tapping and MIRtoolbox analysis is noted (HrRS: $100 \%$ and LrRS: $86 \%$ ) based on their non-significant difference in $\mathrm{HrRS}(\mathrm{p}=0.22)$ and their significant one in $\operatorname{LrRS}(p=0.01$ ) (one-sample test statistical procedure of SPSS 25.0 at $p \leq 0.05$, using as test value the MIRtoolbox tempi, that is $105 \mathrm{bpm}$ for $\mathrm{HrRS}$ and $60 \mathrm{bmp}$ for LrRS). Center and bottom: vertical ground reaction force (GRF) curves for the 10 consecutive finger-tapping cycles of a representative participant in the HrRS (center) and the LrRS (bottom).

mic strength features reported to influence body sway $[7,13,17-$ $19,26,27,39]$. In both musical stimuli, data collection concerned the $70 \mathrm{~s}$ from 2:00 to 3:10 min (voluntary and spontaneous body sway).

For a perceptual tempo estimation [14] before data collection, the participants tapped their index finger on a force plate surface for $30 \mathrm{~s}$ at a 1:1 ratio with the musical beat ( $\vee$ Fig. 2) (2:00-2:30 min in both musical stimuli; they were instructed not to look at their finger to avoid visual interference). The finger tapping tempo differed significantly from the LrRS $(p=0.01)$ but not from the HrRS $(p=0.22)$ one ( $\vee$ Fig. 2$)$. The individual ratings (after data collec- tion, $>$ Fig. 3) indicated the perception of significantly different rhythmic strength indices between $\mathrm{H} r \mathrm{RS}$ and LrRS (pulse clarity, feeling of liking and propensity to move [14, 28]).

\section{Data collection procedure}

The body sway spatio-temporal characteristics were assessed through the center of pressure (CoP) recordings (Kistler force plate $60 \mathrm{~cm} \times 40 \mathrm{~cm} \times 3.5 \mathrm{~cm}$, Type: $9286 \mathrm{AA}$, sampling at $100 \mathrm{~Hz}$, BioWare version 3.2.6 data acquisition and analysis software; Kistler, Winterthur, Switzerland). MATLAB 2019b software (MathWorks, 


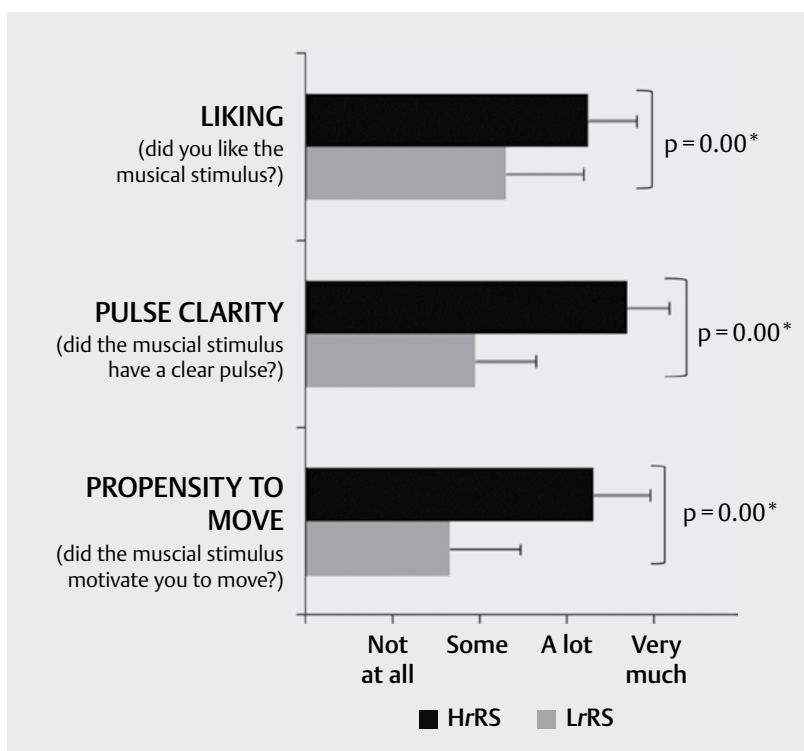

Fig. 3 The mean (SD) of the participants ratings to the 3 item questionnaire regarding their feeling of liking, perception of pulse clarity, and propensity to move for two musical stimuli of higher (HrRS-black bars) and lower (LrRS-grey bars) rhythmic strength (RS). * Significance of difference at $p \leq 0.05$ (Wilcoxon signed-Rank test, SPSS 25.0).

Natick, MA, USA) was used to calculate all variables inserted in statistical analysis.

With their arms along the body and eyes open, the participants performed trials of whole-body voluntary anteroposterior sway (verbal instruction for an ankle-restrained sway, no hip or neck flexion, 70 s data collection), as well as of spontaneous body sway (relaxed upright stance, no instruction to remain as still as possible, 70 s data collection), in three conditions (balanced combination of condition order): without a musical stimulus (non-musical condition, natural sway frequency) and with two musical stimuli (musical conditions, no instruction to synchronize with the musical stimuli, wireless noise-minimizing headphones [40], self-adjusted volume at individual comfort level (60 to $80 \mathrm{~dB})$ ). To minimize any differential effect due to visual information [41] or gaze fixation [42], they were all instructed to fixate their gaze at a standardized target (black cross $6 \times 6 \mathrm{~cm}$, eye level, $2 \mathrm{~m}$ in front of them, natural head posture). To ensure the same feet repositioning across trials, the feet perimeter was traced on a piece of paper secured on the force plate (barefoot to eliminate footwear variance, full contact with the force plate, parallel at preferred width and orientation). A reliability protocol indicated 2 trials as adequate for excellent to fair reliability [43] in the CoP path length across all conditions ( $\vee$ Fig. 4, Table 1s). Previous studies also indicate 2 trials as adequate for excellent reliability $[44,45]$. Thus, 2 trials were decided in all conditions, with 1 min rest between them.

\section{Data analysis}

In the voluntary body sway, the first 5 sways were excluded to avoid the trial initiation transition [28], and the next 10 sways were used for data extraction ( $\vee$ Fig. 5). In the spontaneous body sway, also to avoid the trial initiation transition [28], the first $5 \mathrm{~s}$ were omitted, and the next $60 \mathrm{~s}$ were used for data extraction ( $\triangleright \mathbf{F i g}$. 5). All voluntary body sways demonstrated clear anterior and posterior periodic peaks, and the time between two consecutive anterior peaks defined the single sway duration (s) ( $\bullet$ Fig. 5). After calculating the duration of each one of the 10 retained sways, their mean value defined the sway duration variable (s), and the percentage (\%) of their standard deviation to their mean defined the body sway temporal variability, an estimate of postural control with $[28,46]$ or without $[47,48]$ a musical stimulus.

The following spatial CoP variables were extracted for the voluntary body sway (anteroposterior CoP path of the 10 sways retained for analysis) and the spontaneous one (anteroposterior and mediolateral CoP path during the $60 \mathrm{~s}$ retained for analysis): CoP path length $(\mathrm{cm})$, CoP path variability (\%) (standard deviation of the CoP path length divided by its mean and multiplied by hundred), CoP path range (cm) (anterior to posterior peak distance), CoP path velocity (cm/s) (CoP path length divided by the 10 sways' duration and the 60 s duration, in the voluntary and the spontaneous body sway, respectively), and CoP area $\left(\mathrm{cm}^{2}\right)$ (95\% confidence ellipse fitting). For the voluntary-only body sway, the maximum anterior and posterior CoP displacement (maximum peak, respectively) $(\mathrm{cm})$ was also estimated. For each variable, the two trial averaged value was included in statistics.

\section{Statistical analysis}

Separately in the voluntary and the spontaneous body sway, paired t-tests were used to compare the non-musical condition to each of the musical ones, and the two musical conditions between them (SPSS v.25.0, IBM Corp., Armonk, NY, USA) ( $p \leq 0.05)$. For each variable, the $95 \%$ confidence interval of its mean value in each condition, and the Cohen's d effect size of the between-condition comparisons were also computed.

\section{Results}

In the voluntary ( $\triangleright$ Table 1 ) as well as in the spontaneous ( $\triangleright$ Table 2 ) body sway, none of the CoP variables demonstrated a significant difference between the musical and the non-musical conditions $(p>0.05)$. In the voluntary body sway, the HrRS induced a shorter sway duration ( $p=0.03)$, a longer CoP path $(p=0.04)$, and a higher CoP velocity $(p=0.01)$ compared to the LrRS one ( Table 1$)$, with no significant differences in the spontaneous body sway $(p>0.05)$ ( Table 2).

\section{Discussion}

This study examined if pre-existing musical excerpts among those commonly used in MCS exercise modalities allow the preferred body sway spatio-temporal pattern. The results support our hypothesis and show that indeed, regardless of their rhythmic strength, none of the two musical stimuli altered the preferred voluntary or spontaneous body sway spatio-temporal pattern. The purposeful use of musical stimuli among those commonly used in MCS exercise modalities [14, 21], rather than a casual stimuli selection, denotes an ecological approach that may contribute to external validity. The body sway task that reflects the nature of many 

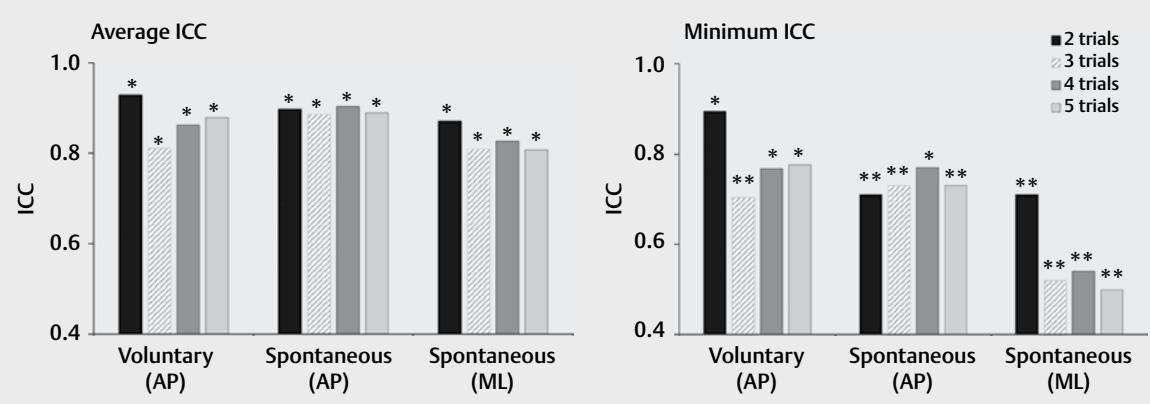

Fig. 4 Average and minimum per body sway intraclass correlation coefficients (ICC) for the CoP path, in the 2, 3, 4, and 5 trials pilot reliability protocol. AP and ML: anteroposterior and mediolateral direction, respectively. Reliability ratings [43]: ${ }^{*}$ Good to excellent: $r>0.75,{ }^{* *}$ moderate to good: $r=0.50-0.75$, small: $r=0.25-0.50$, little or no correlation: $r=0.00-0.25$.

periodic bodyweight shifting or standing (upright, leaning) balance tasks employed in MCS exercise modalities [23,24] may also add to external validity $>$ Table 3.

The main research body focuses on the ability of a musical stimulus to affect body sway (negatively [7-9] or positively $[25,26,28]$ ). Thus, despite the precedent for spontaneous body sway [27, 29], one may be surprised by the rationale concerning the inability of musical stimulus to affect movement. Such variable findings may reflect the inherent redundancy of possible strategies to generate a stable stance that may mask or void the auditory effect [49]. The affordance of a musical stimulus to influence the spatio-temporal body sway measures associates with its features $[7,13,14$, $17,26,27]$. The rhythmic strength features (tempo, pulse clarity, event density, flux, low energy) relate to the level as well as to the directional influence of a musical stimulus $[7,13,26,27]$.

In MCS exercise modalities, a musical accompaniment is considered to obstruct their fundament of preferred movement pace $[5,6]$. However, the association of music with pleasure [15] motivates its use; in such a case, a low tempo/low pulse clarity musical background is suggested [16]. Nevertheless, the relevant recommendations appear extrapolated rather than evidence-based, reasoned on the capacity of rhythmic strength descriptors to increase the propensity to move [17-19]. Such relationships indicate the high pulse clarity as favoring the temporal regulation of periodic movements to the underlying musical pulse periodicities $[13,14,50]$, as well as increasing the amount, speed, and spatial effect of a musical stimulus $[13,14]$. The lower than $100 \mathrm{bpm}$ limit in MCS exercise modalities indicates a tempo slower than the one optimizing the propensity to move (120 bpm) [23,39], most likely due to its coincidence with the well-established natural tempo of human movement in gait [51,52] and finger tapping [53] studies. Tempi lower than 100 bpm may favor movement smoothness, coordination, and stability $[39,54,55]$, most likely because they drive the autonomic nervous system at activation levels lower than the natural ones [20,54]. However, such notions need to be tested in protocols focused on the propensity not to move, rather than inferred from studies on the propensity to move.

The HrRS to LrRS differences in voluntary body sway may relate to the slight exceedance of the $\mathrm{HrRS}$ tempo ( $5 \mathrm{bpm}$ ) over the suggested one for MCS exercise modalities (<100 bpm [16]). Howev- er, it may also indicate that when a similar effect is intended through long-lasting passive listening (as in MCS exercise modalities), the overall rhythmic strength should be considered rather than just slow tempo and low pulse clarity. In this line of thinking, the HrRS' more intense rhythmic features in the low-frequency range most likely underlie its body sway differences with LrRS $[13,46,56,57]$. People appear to align their movements to lowpitched rhythms $[13,46,56]$ independently of movement tempi [46], with the strong rhythmic structures in low frequencies enhancing body movements $[13,56]$.

Interestingly, even though not instructed to sway with the music, the voluntary body sway duration in $\mathrm{HrRS}$ was coupled with the musical phrase ( 8 beats), corroborating earlier work on the relation between metrical levels and the propensity to move [28]. The beat and the bar level are the most essential metrical levels [ 50 , pp. 105-106] for movement association to tempo [39]. In the fitness music industry, the typical metrical structure is a bar consisting of four beats (notated at the quarter-note level), with two bars creating the sense of a musical phrase [16]. Thus, the monotonic relation of inertial load dynamics to natural frequency may explain the coupling of the whole body natural frequency $(0.25 \mathrm{~Hz}$ [25] to a global (2 bars) rather than a local (beat) timescale, as typically occurs when a low inertial load renders a higher natural frequency (i. e., $1.5-2.5 \mathrm{~Hz}$ of finger tapping [58]).

The voluntary body sway results are not without limitations, as these were obtained from an ankle-restrained body rotation rather than a free-form body sway, which could be argued as higher in external validity. However, a free-form body sway not only does not reflect the nature of movements in MCS exercise modalities but could also induce uncontrolled influence on postural stability $[28,45]$. Specifically, the ankle restraint aimed to avoid confounding factors such as both hip and ankle strategy [25], segmental movements (head and upper limb) due to a differential influence of musical features other than tempo and pulse clarity [13], or interparticipant differences concerning the timescale embodiment of pulse periodicities [14]. Such factors may relate to the individual intrinsic variability that appears to differentiate the degree that auditory rhythms (particularly when low-pitched) affect the movement tempo and amplitude (lower/higher influence in more/less intrinsic movement variability) [46]. The suitability of the finger- 


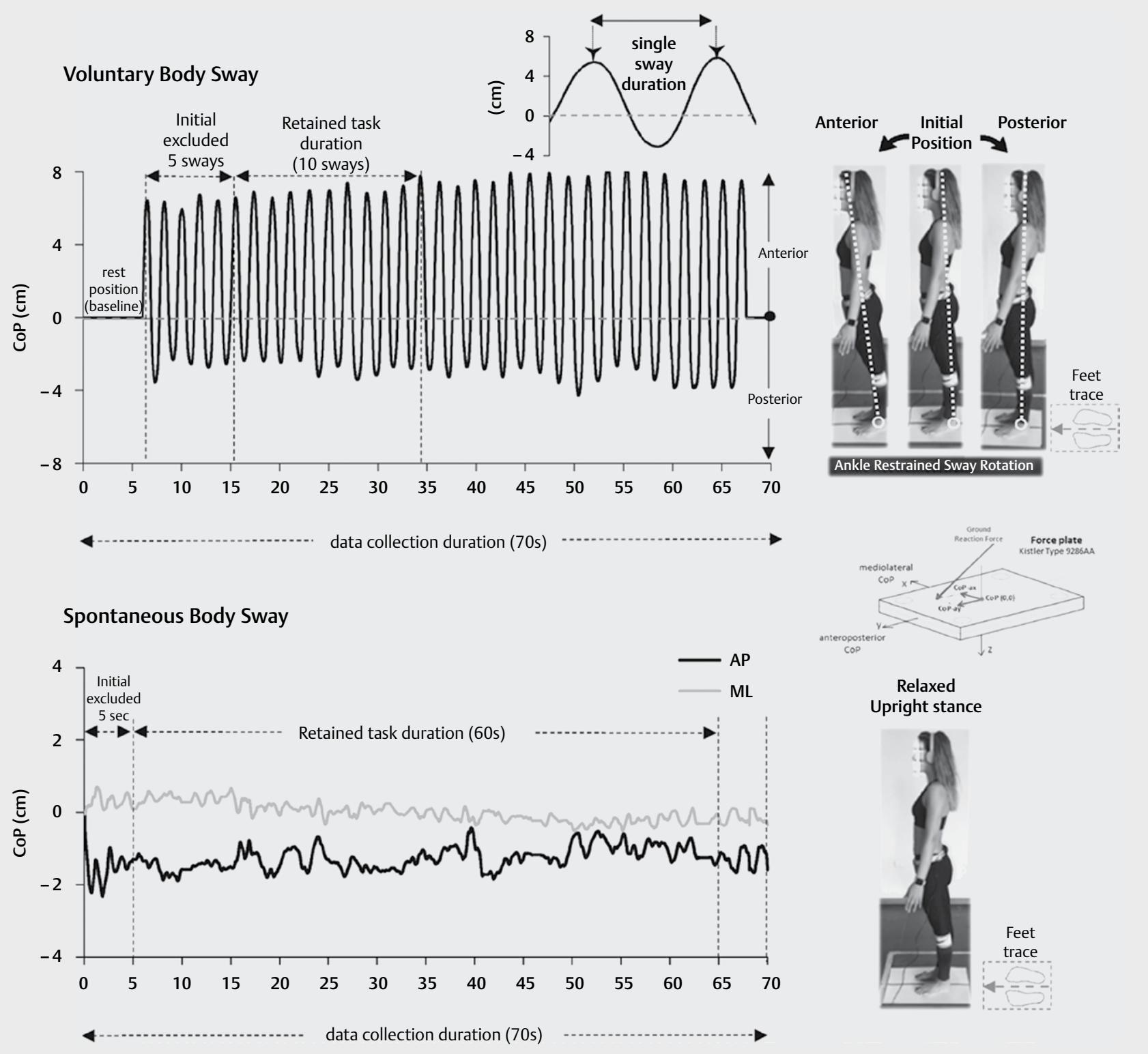

- Fig. 5 Voluntary (top) and spontaneous (bottom) body sway CoP trajectories for a representative participant. The following are indicated for the voluntary and the spontaneous body sway, respectively: the whole data collection duration ( $70 \mathrm{~s}$ in both), and the initial excluded data to avoid the trial initiation transition ( 5 sways and $5 \mathrm{~s}$ ) as well as the retained task duration ( 10 sways and $60 \mathrm{~s}$ ). The ankle-restrained rotation during the voluntary body sway and the relaxed stance during the spontaneous sway are also illustrated by one of the participants.

tapping task to estimate the subjective perception of pulse clarity could also be argued as not having a straightforward relation with whole-body sway due to their distinct natural frequencies [25, 58]. However, a finger tapping test was also used for lateral free-form body sway, which is more complex and of more variable degrees of freedom than the ankle-restrained anteroposterior body sway of this study [14].

In conclusion, the main finding of the present study was that the real, pre-existing musical excerpts commonly used in MCS exercise modalities did not regulate the spatio-temporal pattern of the voluntary and spontaneous body sway away from the preferred one.
Our results provide evidence-based support for the low tempo/low pulse clarity recommendations in MCS exercise modalities, aiming to ensure their fundament for movement learning and performance, citing the preferred movement pace. They also indicate that when a similar effect is intended through long-lasting passive listening (as in MCS exercise modalities), the low-frequency features rather than just tempo and pulse clarity should also be considered. Thus, if instructors familiarize themselves with musical signal analysis, they may purposefully take them into account when selecting a musical accompaniment for MCS exercise modalities. 
- Table 2 Voluntary body sway. Top: Mean \pm SD of the CoP variables for the non-musical stimulus (NM) condition as well as for the two musical stimuli of higher ( $\mathrm{HrRS}$ ) and lower $(\mathrm{HrRS}$ ) rhythmic strength. The significance ( $\mathrm{p}$ values) of the comparisons between conditions is also presented. Bottom: Lower and upper bound of the mean 95\% confidence interval and Cohen's d effect size.

\begin{tabular}{|c|c|c|c|c|c|c|}
\hline Variables & NM & HrRS & HrRS & $\begin{array}{l}\text { NM vs. } \\
\text { HrRS }\end{array}$ & $\begin{array}{l}\text { NM vs. } \\
\text { HrRS }\end{array}$ & $\begin{array}{l}\text { HrRS vs. } \\
\text { LrRS }\end{array}$ \\
\hline & \multicolumn{3}{|c|}{ Mean \pm SD } & \multicolumn{3}{|c|}{ Paired t-tests $p$ values } \\
\hline Sway duration (s) & $4.36 \pm 2.04$ & $4.01 \pm 1.97$ & $4.54 \pm 2.34$ & 0.105 & 0.781 & $0.031^{*}$ \\
\hline Sway duration variability (\%) & $9.44 \pm 2.35$ & $9.18 \pm 2.62$ & $9.50 \pm 2.36$ & 1.000 & 1.000 & 1.000 \\
\hline CoP path $(\mathrm{cm})$ & $288.6 \pm 63.1$ & $295.9 \pm 57.1$ & $282.7 \pm 56.1$ & 0.334 & 1.000 & $0.046^{*}$ \\
\hline CoP path variability (\%) & $54.99 \pm 13.71$ & $55.17 \pm 12.11$ & $55.92 \pm 11.45$ & 1.000 & 1.000 & 1.000 \\
\hline CoP velocity $(\mathrm{cm} / \mathrm{s})$ & $8.19 \pm 2.65$ & $9.13 \pm 2.84$ & $7.90 \pm 2.91$ & 0.055 & 1.000 & $0.012^{*}$ \\
\hline CoP range $(\mathrm{cm})$ & $17.05 \pm 2.74$ & $17.49 \pm 2.40$ & $16.83 \pm 2.77$ & 0.371 & 1.000 & 0.135 \\
\hline CoP area $\left(\mathrm{cm}^{2}\right)$ & $47.34 \pm 15.64$ & $47.84 \pm 19.21$ & $43.99 \pm 16.35$ & 1.000 & 0.406 & 0.937 \\
\hline Maximum anterior CoP displacement $(\mathrm{cm})$ & $10.13 \pm 2.99$ & $10.20 \pm 2.02$ & $10.39 \pm 2.33$ & 1.000 & 1.000 & 1.000 \\
\hline \multirow[t]{2}{*}{ Maximum posterior CoP displacement $(\mathrm{cm})$} & $5.10 \pm 1.80$ & $5.12 \pm 1.73$ & $5.01 \pm 1.66$ & 1.000 & 1.000 & 1.000 \\
\hline & \multicolumn{3}{|c|}{$95 \%$ confidence interval Lower bound-Upper bound } & \multicolumn{3}{|c|}{ Cohen's d effect size } \\
\hline Sway duration (s) & $(3.40-5.31)$ & $(3.1-4.93)$ & $(3.44-5.63)$ & 0.17 & 0.08 & 0.24 \\
\hline Sway duration variability (\%) & $(8.34-10.53)$ & $(7.95-10.41)$ & $(8.4-10.6)$ & 0.10 & 0.03 & 0.13 \\
\hline CoP path $(\mathrm{cm})$ & $(259.0-318.5)$ & $(269.2-322.6)$ & $(256.5-309.0)$ & 0.12 & 0.10 & 0.23 \\
\hline CoP path variability (\%) & $(48.57-61.41)$ & $(49.5-60.84)$ & $(50.56-61.28)$ & 0.01 & 0.07 & 0.06 \\
\hline CoP velocity $(\mathrm{cm} / \mathrm{s})$ & $(6.95-9.43)$ & $(7.81-10.46)$ & $(6.53-9.26)$ & 0.35 & 0.10 & 0.43 \\
\hline CoP range $(\mathrm{cm})$ & $(15.77-18.33)$ & $(16.37-18.61)$ & $(15.53-18.12)$ & 0.17 & 0.08 & 0.26 \\
\hline CoP area $\left(\mathrm{cm}^{2}\right)$ & $(40.02-54.66)$ & $(38.85-56.83)$ & $(36.33-51.64)$ & 0.03 & 0.21 & 0.22 \\
\hline Maximum anterior CoP displacement $(\mathrm{cm})$ & $(8.73-11.53)$ & $(9.26-11.15)$ & $(9.3-11.49)$ & 0.03 & 0.10 & 0.09 \\
\hline Maximum posterior CoP displacement $(\mathrm{cm})$ & $(4.26-5.94)$ & $(4.31-5.93)$ & $(4.23-5.79)$ & 0.01 & 0.05 & 0.07 \\
\hline
\end{tabular}

- Table 3 Spontaneous body sway. Top: Mean \pm SD of the CoP variables for the non-musical stimulus (NM) condition as well as for the two musical stimuli of higher $(\mathrm{HrRS})$ and lower $(\mathrm{H} r \mathrm{RS})$ rhythmic strength. The significance ( $\mathrm{p}$ values) of the comparisons between conditions is also presented. Bottom: Lower and upper bound of the mean 95\% confidence interval and Cohen's d effect size.

\begin{tabular}{|c|c|c|c|c|c|c|c|}
\hline \multicolumn{2}{|l|}{ Variables } & NM & HrRS & HrRS & NM vs. HrRS & NM vs. & HrRS vs. \\
\hline & & \multicolumn{3}{|c|}{ Mean \pm SD } & \multicolumn{3}{|c|}{ Paired t-tests $p$ values } \\
\hline \multirow[t]{2}{*}{ CoP path $(\mathrm{cm})$} & AP & $30.89 \pm 9.44$ & $30.63 \pm 9.13$ & $31.21 \pm 8.09$ & 1.000 & 1.000 & 0.964 \\
\hline & $\mathrm{ML}$ & $20.05 \pm 9.64$ & $18.01 \pm 6.46$ & $19.11 \pm 6.88$ & 0.766 & 1.000 & 0.756 \\
\hline \multirow[t]{2}{*}{ CoP path variability (\%) } & $\mathrm{AP}$ & $15.09 \pm 15.46$ & $13.89 \pm 11.27$ & $19.61 \pm 24.04$ & 1.000 & 0.262 & 0.362 \\
\hline & $\mathrm{ML}$ & $29.38 \pm 18.52$ & $31.31 \pm 22.01$ & $33.74 \pm 26.27$ & 1.000 & 1.000 & 1.000 \\
\hline \multirow[t]{2}{*}{ CoP velocity $(\mathrm{cm} / \mathrm{s})$} & $\mathrm{AP}$ & $0.51 \pm 0.16$ & $0.51 \pm 0.15$ & $0.52 \pm 0.13$ & 1.000 & 1.000 & 0.964 \\
\hline & $\mathrm{ML}$ & $0.33 \pm 0.16$ & $0.30 \pm 0.11$ & $0.32 \pm 0.11$ & 0.766 & 1.000 & 0.756 \\
\hline \multirow[t]{2}{*}{ CoP range $(\mathrm{cm})$} & AP & $2.16 \pm 0.57$ & $2.19 \pm 0.50$ & $2.54 \pm 1.01$ & 1.000 & 0.456 & 0.396 \\
\hline & $\mathrm{ML}$ & $1.25 \pm 0.66$ & $1.26 \pm 0.50$ & $1.28 \pm 0.50$ & 1.000 & 1.000 & 1.000 \\
\hline \multirow[t]{2}{*}{ CoP area $\left(\mathrm{cm}^{2}\right)$} & & $2.37 \pm 2.30$ & $2.26 \pm 1.38$ & $2.33 \pm 1.82$ & 1.000 & 1.000 & 1.000 \\
\hline & & \multicolumn{3}{|c|}{$95 \%$ confidence interval Lower bound-Upper bound } & \multicolumn{3}{|c|}{ Cohen's d effect size } \\
\hline \multirow[t]{2}{*}{ CoP path $(\mathrm{cm})$} & AP & $(26.47-35.31)$ & $(26.35-34.90)$ & $(27.42-35.00)$ & 0.03 & 0.04 & 0.07 \\
\hline & $\mathrm{ML}$ & $(15.54-24.56)$ & $(14.99-21.03)$ & $(15.89-22.33)$ & 0.25 & 0.11 & 0.17 \\
\hline \multirow[t]{2}{*}{ CoP path variability (\%) } & $\mathrm{AP}$ & $(7.85-22.32)$ & $(8.62-19.17)$ & $(8.36-30.87)$ & 0.09 & 0.22 & 0.30 \\
\hline & $\mathrm{ML}$ & $(20.71-38.04)$ & $(21.01-41.61)$ & $(21.45-46.04)$ & 0.10 & 0.19 & 0.10 \\
\hline \multirow[t]{2}{*}{ CoP velocity $(\mathrm{cm} / \mathrm{s})$} & $\mathrm{AP}$ & $(0.44-0.59)$ & $(0.44-0.58)$ & $(0.46-0.58)$ & 0.04 & 0.03 & 0.07 \\
\hline & $\mathrm{ML}$ & $(0.26-0.41)$ & $(0.25-0.35)$ & $(0.26-0.37)$ & 0.24 & 0.10 & 0.17 \\
\hline \multirow[t]{2}{*}{ CoP range $(\mathrm{cm})$} & $\mathrm{AP}$ & $(1.90-2.43)$ & $(1.96-2.43)$ & $(2.07-3.02)$ & 0.05 & 0.46 & 0.44 \\
\hline & $\mathrm{ML}$ & $(0.94-1.56)$ & $(1.04-1.50)$ & $(1.05-1.52)$ & 0.03 & 0.06 & 0.03 \\
\hline CoP area $\left(\mathrm{cm}^{2}\right)$ & & $(1.30-3.45)$ & $(1.62-2.91)$ & $(1.48-3.18)$ & 0.06 & 0.02 & 0.04 \\
\hline
\end{tabular}




\section{Acknowledgements}

We thank George Vagenas, PhD McGill University, Emeritus Professor of Statistics in PESS, School of Physical Education and Sport Science, National and Kapodistrian University of Athens, for his valuable support in the sample power analysis of the study.

\section{Conflict of Interest}

The authors declare that they have no conflict of interest

\section{References}

[1] Karageorghis Cl, Priest DL. Music in the exercise domain: a review and synthesis. Int Rev Sport Exerc Psychol 2012; 5: 44-66

[2] Karageorghis Cl, Priest DL. Music in the exercise domain: A review and synthesis. Int Rev Sport Exerc Psychol 2012; 5: 67-84

[3] Van Dyck E, Leman M. Ergogenic effect of music during running performance. Annals Sport Med Res 2016; 3: 1082

[4] Feiss R, Kostrna J, Scruggs JW et al. Effects of music tempo on perceived exertion, attention, affect, heart rate, and performance during isometric strength exercise. J Sports Sci 2021; 39: 161-169

[5] Pilates HJ, Miller JW. Pilates' Return to Life Through Contrology. Presentation Dynamics; 2012

[6] Isacowitz R, Clippinger K. Pilates Anatomy. Champaign, IL: Human Kinetics; 2020; 2nd Edition.

[7] Gonzalez-Sanchez VE, Zelechowska A, Jensenius AR. Correspondences between music and involuntary human micromotion during standstill. Front Psychol 2018; 9: 1382

[8] Jensenius AR, Zelechowska A, Gonzalez Sanchez VE. The musical influence on people's micromotion when standing still in groups. In: Proceedings of the Sound and Music Computing Conference, July 5-8, Espoo, Finland, 2017; 195-199

[9] Park SH, Lee K, Lockhart T et al. Effects of sound on postural stability during quiet standing. J Neuroeng Rehabil 2011; 8: 67

[10] Arbinaga F, Romero-Pérez N, Torres-Rosado L et al. Influence of music on closed motor skills: a controlled study with novice female dart-throwers. Int J Environ Res Public Health 2020; 17: 4146

[11] Burger B, Toiviainen P. Embodiment in electronic dance music: Effects of musical content and structure on body movement. Musicae Scientiae 2018; 24: 1-20

[12] Blažević I, Vidulin S, Trajkovski B. The efficiency of exercising pilates to music genres. Sport Science 2015; 8: 16-25

[13] Burger B, Thompson MR, Luck $G$ et al. Influences of rhythm- and timbre-related musical features on characteristics of music-induced movement. Front Psychol 2013; 4: 183

[14] Burger B, Thompson MR, Luck $G$ et al. Hunting for the beat in the body: on period and phase locking in music-induced movement. Front Hum Neurosci 2014; 7: 903

[15] Witek MAG, Clarke EF, Wallentin M et al. Syncopation, body-movement and pleasure in groove music. PLoS One 2014; 9: e94446

[16] Kennedy-Armbruster CK, Yoke MM. Methods of Group Exercise Instruction. Human Kinetics, 3rd edition 2014: 60

[17] Burger B, London J, Thompson MR et al. Synchronization to metrical levels in music depends on low-frequency spectral components and tempo. Psychol Res 2017; 82: 1195-1211

[18] Toiviainen P, Burunat I, Brattico E et al. The chronnectome of musical beat. Neuroimage 2020; 216: 116191
[19] Madison G, Gouyon F, Ullén F et al. Modeling the tendency for music to induce movement in humans: first correlations with low-level audio descriptors across music genres. J Exp Psychol Hum Percept Perform 2011; 37: 1578-1594

[20] McPherson T, Berger D, Alagapan S et al. Active and passive rhythmic music therapy interventions differentially modulate sympathetic autonomic nervous system activity. J Music Ther 2019; 56: 240-264

[21] Madison G, Paulin J. Ratings of speed in real music as a function of both original and manipulated beat tempo. J Acoust Soc Am 2010; 128: $3032-3040$

[22] Janata P, Tomic ST, Haberman JM. Sensorimotor coupling in music and the psychology of the groove. J Exp Psychol Gen 2012; 141: 54-75

[23] Page P. Sensorimotor training: A "global" approach for balance training. J Bodyw Mov Ther 2006; 10: 77-84

[24] Freyler K, Krause A, Gollhofer A et al. Specific stimuli induce specific adaptations: sensorimotor training vs. reactive balance training. PLoS One 2016; 11: e0167557

[25] Coste A, Salesse RN, Gueugnon M et al. Standing or swaying to the beat: discrete auditory rhythms entrain stance and promote postural coordination stability. Gait Posture 2018; 59: 28-34

[26] Carrick FR, Oggero E, Pagnacco G. Posturographic changes associated with music listening. J Altern Complement Med 2007; 13: 519-526

[27] Forti S, Filipponi E, Di Berardino F et al. The influence of music on static posturography. J Vestib Res 2010; 20: 351-356

[28] Ross JM, Warlaumont AS, Abney DH et al. Influence of musical groove on postural sway. J Exp Psychol Hum Percept Perform 2016; 42: 308-319

[29] Pagnacco G, Klotzek AS, Carrick FR et al. Effect of tone-based sound stimulation on balance performance of normal subjects: preliminary investigation. Biomed Sci Instrum 2015; 51: 54-61

[30] Vereec KL, Wuyts F, Truijen S et al. Clinical assessment of balance: normative data, and gender and age effects. Int J Audiol 2008; 47: 67-75

[31] Torres SF, Reis JG, Abreu DCC. Influence of gender and physical exercise on balance of healthy young adults. Fisioter Mov 2014; 27: 399-406

[32] Kim J, Kwon Y, Chung HY et al. Relationship between body factors and postural sway during natural standing. Int J Precis Eng Manuf 2012; 13 963-968

[33] Horak FB. Postural orientation and equilibrium: what do we need to know about neural control of balance to prevent falls? Age Ageing 2006; 35: ii7-ii11

[34] Harriss DJ, Macsween A, Atkinson G. Standards for ethics in sport and exercise science research: 2020 update. Int J Sports Med 2019; 40: 813-817

[35] Palm HG, Strobel J, Achatz G et al. The role and interaction of visual and auditory afferents in postural stability. Gait Posture 2009; 30: 328-333

[36] Bigliassi M, Karageorghis Cl, Nowicky AV et al. Effects of auditory distraction on voluntary movements: exploring the underlying mechanisms associated with parallel processing. Psychol Res 2018; 82: 720-733

[37] Adkin AL, Carpenter MG. New insights on emotional contributions to human postural control. Front Neuro 2018; 9: 789

[38] Lartillot O, Toiviainen P. A Matlab toolbox for musical feature extraction from audio. In: Proceedings of the 10th International Conference on Digital Audio Effects, Bordeaux, France 2007; 237: 244

[39] Etani T, Marui A, Kawase $S$ et al. Optimal tempo for groove: its relation to directions of body movement and Japanese nori. Front Psychol 2018; 10: 462

[40] Zelechowska A, Gonzalez-Sanchez VE, Laeng B et al. Headphones or speakers? An exploratory study of their effects on spontaneous body movement to rhythmic music. Front Psychol 2020; 11: 698 
[41] Duarte M, Zatsiorsky V. Effects of body lean and visual information on the equilibrium maintenance during stance. Exp Brain Res 2002; 146: 60-69

[42] Fiorelli C, Polastri P, Rodrigues S et al. Gaze position interferes in body sway in young adults. Neurosci Lett 2017; 660: 130-134

[43] Portney LG, Watkins MP. Foundations of Clinical Research: Applications to Practice. London: Pearson; 2009: 585-616

[44] Lafond D, Corriveau H, Hébert R et al. Intrasession reliability of center of pressure measures of postural steadiness in healthy elderly people. Arch Phys Med Rehabil 2004; 85: 896-901

[45] Raymakers J, Samson M, Verhaar H. The assessment of body sway and the choice of the stability parameters. Gait Posture 2005; 21: 48-58

[46] Varlet M, Williams R, Keller PE. Effects of pitch and tempo of auditory rhythms on spontaneous movement entrainment and stabilization. Psychol Res 2020; 84: 568-584

[47] Palmieri RM, Ingersoll CD, Stone MB et al. Center-of-pressure parameters used in the assessment of postural control. J Sport Rehabil 2002; 11: 51-66

[48] Latash ML, Ferreira SS, Wieczorek SA et al. Movement sway: changes in postural sway during voluntary shifts of the center of pressure. Exp Brain Res 2003; 150: 314-324

[49] Lubetzky AV, Gospodarek M, Arie L et al. Auditory input and postural control in adults. JAMA Otolaryngol Head Neck Surg 2020; 146: 480-487

[50] Hainsworth S. Beat Tracking and Musical Metre Analysis. In: Klapuri A, Davy M.eds. Signal Processing Methods for Music Transcription. Boston: Springer; 2006: 101-129
[51] MacDougall HG, Moore ST. Marching to the beat of the same drummer: the spontaneous tempo of human locomotion. J Appl Physiol 2005; 99: 1164-1173

[52] Styns F, van Noorden L, Moelants D et al. Walking on music. Hum Mov Sci 2007; 26: 769-785

[53] Moelants D. Preferred tempo reconsidered. In: Stevens C, Burnham D, McPherson G. et al. eds. Proceedings of the 7th International Conference on Music Perception and Cognition. Sydney: Causal Productions; 2002: 580-583

[54] Bretherton B, Deuchars J, Windsor WL. The effects of controlled tempo manipulations on cardiovascular autonomic function. Music Science 2019; 2: 1-14

[55] Husain G, Thompson WF, Schellenberg EG. Effects of musical tempo and mode on arousal, mood, and spatial abilities. Music Percept 2002; 20: $151-171$

[56] Hove MJ, Marie C, Bruce IC et al. Superior time perception for lower musical pitch explains why bass-ranged instruments lay down musical rhythms. Proc Natl Acad Sci 2014; 111: 10383-10388

[57] Lenc T, Keller PE, Varlet $M$ et al. Neural tracking of the musical beat is enhanced by low-frequency sounds. Proc Natl Acad Sci 2018; 115: $8221-8226$

[58] Repp BH. Sensorimotor synchronization: A review of the tapping literature. Psychon Bull Rev 2005; 12: 969-992 